\title{
High Prevalence of Sarcopenia in HIV-Infected Individuals
}

\author{
Patricia Echeverría (D), ${ }^{1,2}$ Anna Bonjoch (D), ${ }^{1,2}$ Jordi Puig (D), ${ }^{1}$ Carla Estany, \\ Arelly Ornelas, ${ }^{3}$ Bonaventura Clotet, ${ }^{1,2,4,5}$ and Eugenia Negredo $\mathbb{D}^{1,2,5}$ \\ ${ }^{1}$ Lluita contra la Sida Foundation, Hospital Universitari Germans Trias i Pujol, Badalona, Spain \\ ${ }^{2}$ Universitat Autònoma de Barcelona, Catalonia 08916, Spain \\ ${ }^{3}$ Statistics and Operations Research, Technical University of Catalunya, Barcelona 08020, Spain \\ ${ }^{4}$ AIDS Research Institute-IRSICAIXA, Institut Germans Trias I Pujol (IGTP), Hospital Universitari Germans Trias i Pujol, \\ Badalona 08916, Spain \\ ${ }^{5}$ Universitat de Vic-Universidad Central de Catalunya UVIC-UCC, Vic 08500, Spain
}

Correspondence should be addressed to Patricia Echeverría; pecheverria@flsida.org

Received 4 April 2018; Revised 15 June 2018; Accepted 26 June 2018; Published 12 July 2018

Academic Editor: Giulia Morsica

Copyright (C) 2018 Patricia Echeverría et al. This is an open access article distributed under the Creative Commons Attribution License, which permits unrestricted use, distribution, and reproduction in any medium, provided the original work is properly cited.

\begin{abstract}
Background. Sarcopenia is a geriatric syndrome that leads to a loss of functionality and mortality. Methods. We assessed the prevalence of sarcopenia in HIV-infected patients attended in our HIV Unit who had at least two DXA scans from 2000 to 2016 (1,720 DXA scans from 860 individuals). Sarcopenia was determinate according to appendicular skeletal muscle mass index (ASM) calculated as the ratio between skeletal muscle mass index (SMI) by DXA and height ${ }^{2}\left(\mathrm{~kg} / \mathrm{m}^{2}\right)$. We stratified patients by gender and age $(<40,41-50$, and $>50$ years) and according to the interval between DXAs $(\leq 3,3-7,7-10,>10$ years). The statistical analysis was performed using SPSS version 19. Results. Median (IQR) age was $52(47 ; 57)$ years, and $76 \%$ were male. The median (IQR) time with HIV infection was $8(3 ; 15)$ years. The prevalence of sarcopenia was $25.7 \%$ (95\% CI, 22.8-28.7), more prevalent in those aged $>50$ years $(27.8 \%)$. Stratifying by gender, $43 \%$ of women aged $>50$ years presented sarcopenia compared with $8.8 \%$ of men. The frequency of sarcopenia increased from $37.6 \%$ to $49.4 \%$ when interval between DXA was 7-10 years $(n=109)$, significantly higher in women than in men $(\mathrm{p}=0.016)$. In addition to the traditional risk factors, time with HIV infection was associated with sarcopenia [RR 1.780 (95\% CI, 1.314-2.411), $\mathrm{p}=0.001]$. Conclusion. The prevalence and progression of sarcopenia in HIV-infected patients were high, mainly among women. Further studies are necessary to assess the best approaches to prevent this condition and its consequences.
\end{abstract}

\section{Introduction}

The enormous advances in antiretroviral treatment in the last two decades have made infection by human immunodeficiency virus (HIV) in developed countries to be considered a chronic disease. However, as a consequence of the improvement in survival, the prevalence of comorbidities is increasing, as shown by data from large observational cohorts of HIV-infected patients [1-3]. In addition, some studies suggest that HIV-related chronic inflammation and longterm exposure to some antiretroviral drugs are responsible for accentuated aging in this population [4]. Consequently, physicians are increasingly faced with previously unrecognized comorbid conditions in HIV-infected patients $[1-3,5]$.
There is increasing evidence that the higher prevalence of bone fractures in HIV-infected individuals may lead to immobility, dependency, and increased morbidity and mortality [6-10]. Although increased bone mass loss is a crucial factor for bone fractures, the presence of sarcopenia (from the Greek sarx, or flesh, and penia, loss) could contribute to an increased risk of falls and fractures (directly or increasing the frailty) [11].

Sarcopenia is a syndrome characterized by progressive and generalized loss of skeletal muscle mass and strength that has been associated with a risk of adverse outcomes such as physical disability, poor quality of life, morbidity, and death. It is highly prevalent in older populations $[12,13]$. 
The European Working Group on Sarcopenia in Older People (EWGSOP) developed a practical clinical definition and consensus diagnostic criteria for age-related sarcopenia [14]. Previous cross-sectional studies in Europe and North America have described a sarcopenia index (muscle mass/ height ${ }^{2}$ ) and used a cut-off based on two standard deviations (2SD) below mean of young adults $[15,16]$. However, sarcopenia definition is currently a matter of debate and several new indices are being tested [14-19].

The most widely used imaging techniques for estimating muscle mass and lean body mass are computed tomography (CT), magnetic resonance imaging (MRI), and dual-energy $\mathrm{X}$-ray absorptiometry (DXA) [14, 15]. DXA is an attractive alternative method for research and for clinical use in the analysis of body composition, providing assessment and quantification of fat mass, lean mass, and bone mineral content, especially through the assessment of nonbone lean mass parameters, such as appendicular skeletal lean mass (ASM) adjusted for body mass index (BMI) and height (ASM/BMI and $\mathrm{ASM} / \mathrm{ht}^{2}$ ). ASM, which is a sum of the muscle mass of the arms and legs, is generally used for the skeletal muscle mass index and distinguishes individuals with physiological loss of muscle mass from those with pathological worsening of sarcopenia [14-16].

The multiple factors that contribute to sarcopenia include the aging process, suboptimal diet and bed rest, sedentary lifestyle, chronic diseases, and certain drug treatments [13, $14]$.

Considering the high relevance of sarcopenia in elderly people, this study aims to determine the prevalence of sarcopenia (determinate by DXA scan) and its progression in a cohort of HIV-1-infected subjects. The study also assesses the relationship between sarcopenia and other HIV-related characteristics and clinical factors.

\section{Methods}

2.1. Study Design and Population. This analysis was based on DXA scans from all HIV-infected individuals attended in the HIV Unit of a tertiary hospital who had at least two DXA scans over the last 17 years (from 2000 to 2016). The DXA scans (LUNAR DPX-L, Madison, WI, USA) were requested as part of the patient's follow-up in clinical practice or in the context of clinical trials, and since 2015, DXA scans were requested according to the current recommendations for HIV-infected persons: before starting antiretroviral treatment, men aged $>50$ years old, postmenopausal women, persons with a history of fractures, and patients with diseases associated with a decrease in bone mineral density. [20]

Sarcopenia was determinate according to appendicular skeletal muscle mass index (ASM) calculated as the ratio between skeletal muscle mass index (SMI) by DXA and height $^{2}\left(\mathrm{~kg} / \mathrm{m}^{2}\right)$ [15]. The cut-off point used was two standard deviations (2SD) below the mean SMI of young male and female reference groups. Patients were considered to have sarcopenia when the SMI was $5.5 \mathrm{~kg} / \mathrm{m}^{2}$ in women and 7.26 $\mathrm{kg} / \mathrm{m}^{2}$ in men $[14,15]$.
2.2. Study Endpoint. The prevalence of sarcopenia was determined in the overall population and stratified by gender and age (<40, 41-50, and $>50$ years).

Progression of sarcopenia was defined as the difference between the baseline prevalence registered in the first DXA scan and the prevalence calculated in the last DXA scan available for each patient. Patients were stratified according to gender and age, taking into account the interval between the first and the last DXA scan ( $<3$ years, 3-7 years, 7-10 years, and $>10$ years).

The factors assessed to determine the predisposing risk factors for sarcopenia were gender, age, BMD, body mass index (BMI), specific HIV-related characteristics (time since diagnosis of HIV, cumulative exposure to antiretroviral treatments, CD4 cell count, and viral load) and other clinical parameters (cholesterol levels, osteopenia/osteoporosis), adjusted for gender and age.

Sociodemographic data, HIV-related data, and anthropometric and clinical data were obtained from the medical records.

2.3. Statistical Analysis. Demographic and clinical parameters were expressed as the mean and standard deviation (SD) or as the median and interquartile range (IQR); qualitative variables were expressed as frequencies and percentages. Normally distributed continuous variables were compared using the $t$-test; nonnormally distributed variables were compared using the Mann-Whitney test. The dependent $t$ test for paired samples or the Wilcoxon signed rank test was performed to assess the significance of changes observed over time; proportions were compared using the McNemar test.

Univariate $P$ values $<0.05$ were considered significant.

The statistical analysis was performed using IBM SPSS Statistics for Windows, Version 19.0 (IBM Corp, Armonk, New York, USA).

\section{Results}

A total of 1,720 DXA scans from $860 \mathrm{HIV}$-1-infected individuals were analysed. At time of the first DXA scan, the median (IQR) age of the population was $52(47 ; 57)$ years; men accounted for $76 \%$ of the study population. The median (IQR) time since the diagnosis of HIV infection was $8(3 ; 15)$ years, and the cumulative exposure to antiretroviral treatment was $8(3 ; 14)$ years. Almost all patients $(94 \%)$ had an HIV-1 RNA viral load $\leq 50$ copies/ml and a median (IQR) CD4 T-cell count of $654(472 ; 874)$ cells $/ \mathrm{mm}^{3}$ (Table 1$)$.

3.1. Prevalence of Sarcopenia. The prevalence of sarcopenia overall (according to the parameters evaluated in the DXA scans included in the analysis) was $25.7 \%$ (95\% CI: 22.8-28.7) (Table 2).

Sarcopenia was more prevalent in patients aged 41-50 years $(40 \%)$ and $>50$ years $(59 \%)$ (Table 2$)$.

When sarcopenia was assessed according to age and gender, $43 \%$ of women older than 50 years and $8.8 \%$ of men had sarcopenia $(\mathrm{p}=0.001)$ (Table 2 ).

The percentage of sarcopenia in patients with osteopenia and osteoporosis was $34 \%$ and $10.5 \%$, respectively. Sarcopenia 
TABLE 1: Clinical and epidemiological characteristics of the study population at time of the first DXA scan.

\begin{tabular}{|c|c|}
\hline & $\mathrm{N}=\mathbf{8 6 0}$ \\
\hline Age, years (median [IQR]) & $52(47 ; 57)$ \\
\hline Age $\geq 50$ years $(\%)$ & 59 \\
\hline Gender (male) (\%) & 76 \\
\hline Time since diagnosis of HIV infection, years (median [IQR]) & $8(3 ; 15)$ \\
\hline Cumulative exposure to antiretrovirals, years (median [IQR]) & $8(3 ; 14)$ \\
\hline CD4 cell count $/ \mu \mathrm{L}$ (median [IQR]) (baseline) & $552(377 ; 728)$ \\
\hline HIV-RNA $\leq 50$ copies/mL (\%) & 94 \\
\hline Osteopenia/osteoporosis (\%) & $51 / 18$ \\
\hline Body mass index (median [IQR]) & $23(21 ; 25)$ \\
\hline Total cholesterol (mmol/ml) (median [IQR]) & $4.7(4 ; 5.7)$ \\
\hline
\end{tabular}

TABle 2: Prevalence of sarcopenia in HIV-infected persons.

\begin{tabular}{|c|c|c|c|c|c|c|}
\hline \multicolumn{7}{|c|}{ Prevalence of sarcopenia according to gender } \\
\hline & \multicolumn{3}{|c|}{ First DXA } & \multicolumn{3}{|c|}{ Last DXA } \\
\hline & $\begin{array}{c}\text { Female }^{*} \\
(\mathrm{n}=210)\end{array}$ & $\begin{array}{l}\text { Male }^{* *} \\
(\mathrm{n}=650)\end{array}$ & P value & $\begin{array}{c}\text { Female }^{*} \\
(\mathrm{n}=210)\end{array}$ & $\begin{array}{c}\text { Male }^{* *} \\
(\mathrm{n}=650)\end{array}$ & P value \\
\hline No sarcopenia (\%) & 35 & 70 & $<0.001$ & 43 & 73 & $<0.001$ \\
\hline Sarcopenia (\%) & 65 & 30 & $<0.001$ & 57 & 27 & $<0.001$ \\
\hline \multicolumn{7}{|c|}{ Prevalence of sarcopenia according to age } \\
\hline$\leq 40$ years $(\%)$ & & & & 1 & & \\
\hline $41-50$ years $(\%)$ & & & & 40 & & \\
\hline$>50$ years $(\%)$ & & & & 59 & & \\
\hline \multicolumn{7}{|c|}{ Prevalence of sarcopenia according to gender and age $\geq 50$ years } \\
\hline $\mathrm{N}=\mathbf{5 0 7}$ & & $\begin{aligned} \text { Female } & \geq \mathbf{5 0} \text { years } \\
\mathrm{n} & =129\end{aligned}$ & & & $\begin{array}{c}\text { Male }>\mathbf{5 0} \text { years } \\
\mathrm{n}=378\end{array}$ & \\
\hline Sarcopenia n (\%) & & $55(43)$ & & & $33(8.8)$ & \\
\hline
\end{tabular}

was not observed in patients with a normal BMD (55\%) $(\mathrm{p}=0.001)$.

3.2. Progression of Sarcopenia and Risk Factors. The frequency of sarcopenia increased from $37.6 \%$ to $49.4 \%$ $(p=0.001)$ in patients with an interval of 7-10 years between DXA scans ( $\mathrm{n}=109$ patients) and from $22 \%$ to $25.4 \%$ $(p=0.046)$ in those with an interval of more than 10 years between DXA scans $(n=209)$.

When the progression of sarcopenia was determined taking into account gender, the overall prevalence increased from $56 \%$ to $66 \%$ in women $(\mathrm{p}=0.016)$ and from $26.7 \%$ to $29.7 \%$ in men $(p=0.057)$, with significant differences between the genders $(\mathrm{p}=0.001)$.

When age was taken into account, the percentage of sarcopenia in individuals older than 50 years increased from $43 \%$ to $52 \%$ in women ( $\mathrm{p}=0.001$ ) and from $8.8 \%$ to $9 \%$ in men $(\mathrm{p}=0.422)$. The difference between the genders was significant $(\mathrm{p}=0.001)$.

The univariate analysis showed that sarcopenia was more prevalent in women than in men [RR 4.502 (95\% CI: 3.227$6.281), \mathrm{p}=0.001$ ], in patients with osteopenia [RR $0.415(95 \%$
CI: 0.303-0.568), $\mathrm{p}<0.001$ ], and in patients with osteoporosis [RR 0.237 (95\% CI: 0.151-0.374), p<0.001].

Multiple regression analysis adjusted for age and gender revealed associations between sarcopenia and other clinical and epidemiological variables included in the analysis (Table 3), such as time with HIV infection [RR $1.780(95 \%$ CI: 1.314-2.411), $\mathrm{p}=0.001]$. However, no associations were observed between HIV-RNA viral load $>50$ copies $/ \mathrm{mL}$ and sarcopenia [R 0.805 (95\% CI: 0.610-1,062), p=0.125], neither between CD4 T-cell counts and sarcopenia [R 0.816 (95\% CI: $0.568-1,172), \mathrm{p}=0.271]$.

\section{Discussion}

In our study, sarcopenia affected one-fifth of the population and significantly increased over time, being more prevalent in women older than 50 years. Longer duration of HIV infection was associated with a greater risk of sarcopenia.

Based on DXA scans, we found a high prevalence of sarcopenia in HIV-infected individuals (25\%). This finding is similar to rates reported in longitudinal and other crosssectional studies in HIV-infected persons after adjusting for age, gender, and BMI [11, 21]. 
TABLE 3: Multivariate adjusted logistic regression analysis.

\begin{tabular}{lcr}
\hline & RR (95\% CI) & Adjusted $^{\text {a }}$ \\
\hline Age (30-50 years) & $3.26(0.928-11.505)$ & P value \\
\hline Gender (female vs male) & $4.502(3.227-6.281)$ & $<0.001$ \\
\hline Time since diagnosis of HIV infection $>5$ years & $1.780(1.314-2.411)$ & $<0.001$ \\
\hline Body mass index $>20$ & $0.194(0.076-0.497)$ & $<0.001$ \\
\hline Total cholesterol $<140 \mathrm{mg} / \mathrm{dL}$ & $1.468(0.949-2.271)$ & $<0.001$ \\
\hline
\end{tabular}

${ }^{\mathrm{a}}$ The final model of multivariate analyses was adjusted for the following predictors: age, gender, time of diagnosis of HIV-infection $>5$ years, BMI $>20$, viral load $\geq 50$ copies $/ \mathrm{mL}$, and total cholesterol $<140 \mathrm{mg} / \mathrm{dL}$.

Ferreira Da Silva et al. [11] evaluated the presence of presarcopenia and sarcopenia in HIV-infected individuals and compared a group of virologically suppressed patients receiving regular antiretroviral therapy and elderly non-HIVinfected controls. Consistent with our results, the authors showed a strong positive association between presarcopenia and sarcopenia in persons with HIV infection, even after taking into account the higher mean age of controls (59 versus 70 years) and adjusting for age and BMI.

Similarly, Wasserman et al. [21] reported a prevalence of low muscle mass similar to ours (between $18.8 \%$ and $21.9 \%$, depending on the definition used) in midlife and older HIV-infected individuals, particularly men, despite CD4 cell reconstitution and viral suppression. However, in our analysis, when we considered only males, we found a lower prevalence of sarcopenia. [11, 21] Yarasheski et al. [22] found similar data in a longitudinal study that also included a non-HIV-infected control group.

The small sample size of the previous studies, however, makes it difficult to stratify patients by gender and age. Our large sample, on the other hand, enabled us to assess differences between men and women of different ages and identify a higher prevalence among women, mainly those older than 50 years.

The prevalence of sarcopenia clearly varies according to which diagnostic criteria are applied to different study samples.

As expected, progression of sarcopenia was more pronounced in patients with an interval of $\geq 7$ years between DXAs. Women aged $\geq 50$ years presented more severe sarcopenia. Although data on progression are comparable to findings for other HIV-infected cohorts [11, 21-23], no previous studies have taken gender into account.

Our results confirm that the traditional risk factors for sarcopenia in HIV-infected population are the same as in the general population. Older age, female gender, and low BMI and low cholesterol were associated with low muscle mass [22]. In addition to the traditional risk factors, however, other HIV-related factors could play an important role in the development of sarcopenia in this population. As our study shows, longer time with HIV infection predisposed to muscle mass loss, thus potentially explaining the higher prevalence than in noninfected controls, as confirmed in the previously mentioned studies (20\% in HIV-infected persons versus $11 \%-28 \%$ in non-HIV-infected persons). In contrast, CD4 cell count and viral suppression were not associated factors. The fact that most patients had an undetectable viral load prevented us from assessing the role of viral replication on muscle mass loss.

The retrospective design of our study was not possible to determine the impact of some risk factors (as smoking, alcohol use, physical activity, or antiretroviral treatment) on the development of sarcopenia, due to the lack of necessary information on the medical report. Prospective studies are necessary (which included a non-HIV-infected control group) to compare the prevalence of sarcopenia between HIV-infected subjects and non-HIV population.

Although we were able to draw robust conclusions especially in postmenopausal women owing mainly to the huge number of DXA scans $(1,720$ scans from 860 HIV-infected individuals), the wide age range (with 507 patients aged $\geq 50$ years), and the high rate of women included (a quarter of the population).

Therefore, given the prevalence and progression of sarcopenia over time and sarcopenia-associated consequences such as fragility, disability, and mortality [11, 21-23], physicians should consider this syndrome in vulnerable persons, mainly postmenopausal women, to prevent progression and its clinical consequences.

In summary, this exploratory analysis shows a high prevalence of sarcopenia among HIV-infected persons, particularly women, and a positive association between sarcopenia and time with HIV infection. Future research should determine the impact of sarcopenia on morbidity, physical function, and quality of life in HIV-infected individuals and develop approaches to prevent the condition.

\section{Data Availability}

This data is available in the following link: http://www.natap .org/2017/AdverseReactComor/AdverseReactComor_15.htm. The data used to support the findings of this study are available from the corresponding author upon request.

\section{Disclosure}

The work has been presented in $19^{\text {th }}$ International Workshop on Co-Morbidities and Adverse Drug Reactions; $16^{\text {th }}$ European AIDS Conference (EACS), 25-27 October, 2017 (Milan, Italy), and has not been published or considered for publication elsewhere. 


\section{Conflicts of Interest}

None of the authors has a financial or other interest in the concepts mentioned in the present article.

\section{Authors' Contributions}

Patricia Echeverría, Anna Bonjoch, Jordi Puig, Bonaventura Clotet, and Eugenia Negredo participated in the study design, patient selection, data analysis, and critical review of the manuscript. Arelly Ornelas conducted statistical analyses. All authors read and approved the final manuscript.

\section{Acknowledgments}

This work was supported by "Lluita contra la SIDA" Foundation. Partial results of this study were presented as an "oral presentation" in $19^{\text {th }}$ International Workshop on CoMorbidities and Adverse Drug Reactions; $16^{\text {th }}$ European AIDS Conference (EACS), 25-27 October, 2017 (Milan, Italy). This work was supported by "Lluita contra la SIDA" Foundation.

\section{References}

[1] S. G. Deeks and A. N. Phillips, "HIV infection, antiretroviral treatment, ageing, and non-AIDS related morbidity," BMJ, vol. 338, no. 3172, 2009.

[2] S. G. Deeks, "HIV infection, inflammation, immunosenescence, and aging," Annual Review of Medicine, vol. 62, pp. 141155, 2011.

[3] G. Guaraldi, G. Orlando, S. Zona et al., "Premature age-related comorbidities among HIV-infected persons compared with the general populationa," Clinical Infectious Diseases, vol. 53, no. 11, pp. 1120-1126, 2011.

[4] S. Pathai, H. Bajillan, A. L. Landay, and K. P. High, "Is HIV a model of accelerated or accentuated aging?" The Journals of Gerontology. Series A, Biological Sciences and Medical Sciences, vol. 69, no. 7, pp. 833-842, 2014.

[5] J. B. Kirk and M. B. Goetz, "Human immunodeficiency virus in an aging population, a complication of success," Journal of the American Geriatrics Society, vol. 57, no. 11, pp. 2129-2138, 2009.

[6] L. Gedmintas, E. A. Wright, Y. Dong et al., "Factors associated with fractures in HIV-infected persons: which factors matter?" Osteoporosis International, vol. 28, no. 1, pp. 239-244, 2017.

[7] R. Güerri-Fernández, J. Villar-García, A. Díez-Pérez, and D. Prieto-Alhambra, "HIV infection, bone metabolism, and fractures," Arquivos Brasileiros de Endocrinologia \& Metabologia, vol. 58, no. 5, pp. 478-483, 2014.

[8] B. Young, C. N. Dao, K. Buchacz, R. Baker, and J. T. Brooks, "Increased rates of bone fracture among HIV-infected persons in the HIV outpatient study (HOPS) compared with the US general population, 2000-2006," Clinical Infectious Diseases, vol. 52, no. 8, pp. 1061-1068, 2011.

[9] Á. H. Borges, J. Hoy, E. Florence et al., "Antiretrovirals, Fractures, and Osteonecrosis in a Large International HIV Cohort," Clinical Infectious Diseases, vol. 64, no. 10, pp. 1413-1421, 2017.

[10] J. Hoy and B. Young, "Do people with HIV infection have a higher risk of fracture compared with those without HIV infection?" Current Opinion in HIV and AIDS, vol. 11, no. 3, pp. 301-305, 2016.

[11] L. F. D. S. Pinto Neto, M. C. Sales, E. S. Scaramussa, C. J. C. da Paz, and R. L. Morelato, "Human immunodeficiency virus infection and its association with sarcopenia," The Brazilian Journal of Infectious Diseases, vol. 20, no. 1, pp. 99-102, 2016.

[12] J. E. Morley, R. N. Baumgartner, R. Roubenoff, J. Mayer, and K. S. Nair, "Sarcopenia," Journal of Laboratory and Clinical Medicine, vol. 137, no. 4, pp. 231-243, 2001.

[13] M. Iannuzzi-Sucich, K. M. Prestwood, and A. M. Kenny, "Prevalence of sarcopenia and predictors of skeletal muscle mass in healthy, older men and women," The Journals of Gerontology. Series A, Biological Sciences and Medical Sciences, vol. 57, no. 12, pp. M772-M777, 2002.

[14] A. J. Cruz-Jentoft, J. P. Baeyens, J. M. Bauer et al., "Sarcopenia: European consensus on definition and diagnosis," Age and Ageing, vol. 39, no. 4, Article ID afq034, pp. 412-423, 2010.

[15] R. N. Baumgartner, K. M. Koehler, D. Gallagher et al., "Epidemiology of sarcopenia among the elderly in New Mexico," American Journal of Epidemiology, vol. 147, no. 8, pp. 755-763, 1998.

[16] M. J. Delmonico, T. B. Harris, J. Lee et al., "Alternative definitions of sarcopenia, lower extremity performance, and functional impairment with aging in older men and women," Journal of the American Geriatrics Society, vol. 55, no. 5, pp. 769774, 2007.

[17] I. Janssen, R. N. Baumgartner, R. Ross, I. H. Rosenberg, and R. Roubenoff, "Skeletal muscle cutpoints associated with elevated physical disability risk in older men and women," American Journal of Epidemiology, vol. 159, no. 4, pp. 413-421, 2004.

[18] I. Janssen, S. B. Heymsfield, R. N. Baumgartner, and R. Ross, "Estimation of skeletal muscle mass by bioelectrical impedance analysis," Journal of Applied Physiology, vol. 89, no. 2, pp. 465471, 2000.

[19] J. E. Morley, “Sarcopenia: Diagnosis and treatment," The Journal of Nutrition, Health \& Aging, vol. 12, no. 7, pp. 452-456, 2008.

[20] E. Negredo, P. Domingo, F. Gutiérrez et al., "Executive summary of the consensus document on osteoporosis in HIV-infected individuals," Enfermedades Infecciosas y Microbiología Clínica, vol. 36, no. 5, pp. 312-314, 2018.

[21] P. Wasserman, S. Segal-Maurer, and D. S. Rubin, "High prevalence of low skeletal muscle mass associated with male gender in midlife and older HIV-infected persons despite cd4 cell reconstitution and viral suppression," Journal of the International Association of Providers of AIDS Care, vol. 13, no. 2, pp. 145-152, 2014.

[22] K. E. Yarasheski, R. Scherzer, D. P. Kotler et al., "Age-Related Skeletal Muscle Decline Is Similar in HIV-Infected and Uninfected Individuals," The Journals of Gerontology. Series A, Biological Sciences and Medical Sciences, vol. 66A, no. 3, pp. 332-340, 2011.

[23] L. Desquilbet, J. B. Margolick, L. P. Fried et al., "Relationship between a frailty-related phenotype and progressive deterioration of the immune system in HIV-infected men," Journal of Acquired Immune Deficiency Syndromes, vol. 50, no. 3, pp. 299306, 2009. 


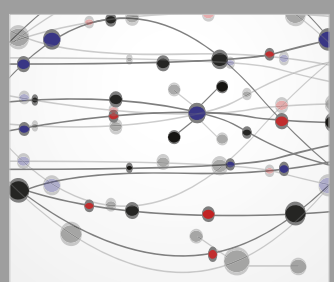

The Scientific World Journal
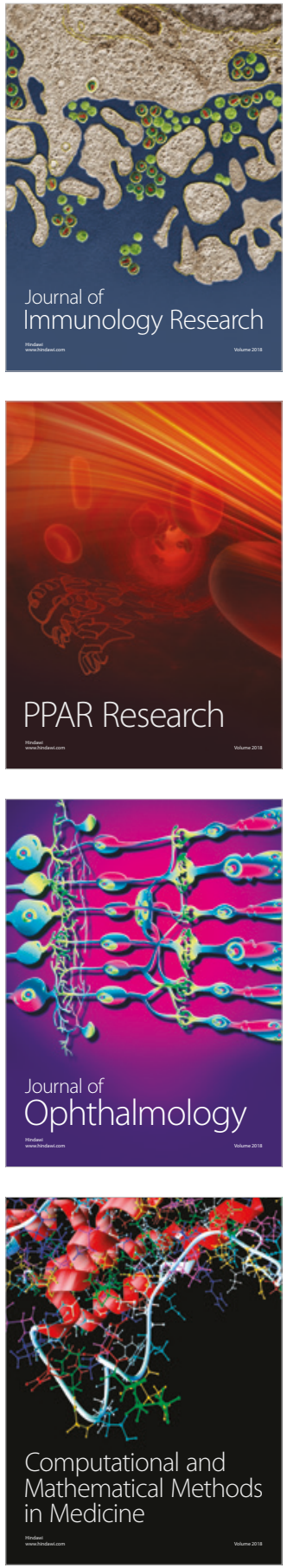

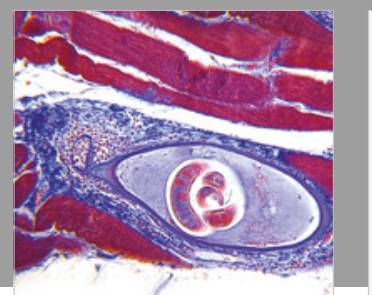

Gastroenterology Research and Practice

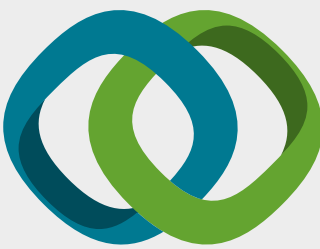

\section{Hindawi}

Submit your manuscripts at

www.hindawi.com
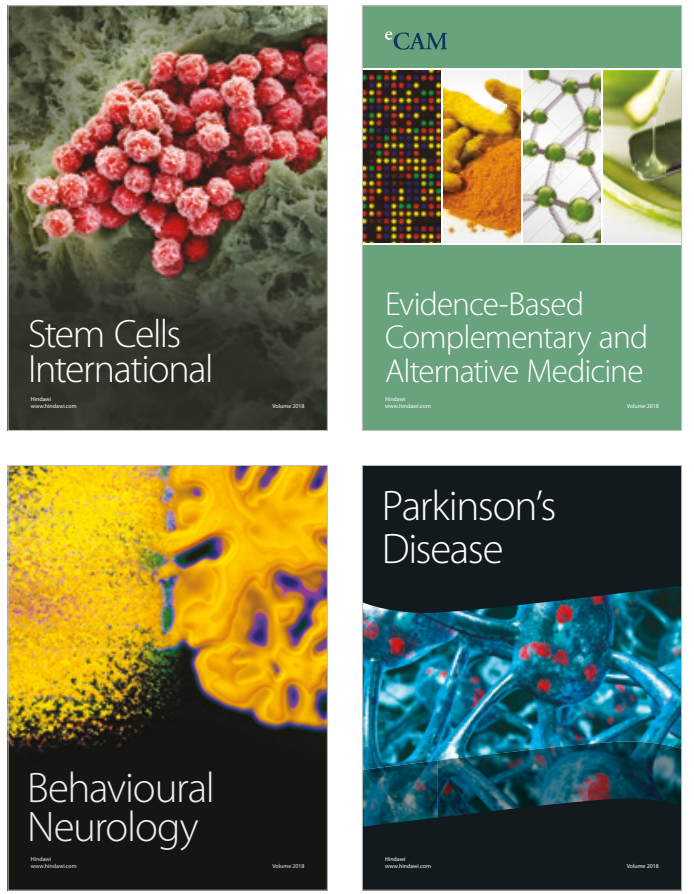

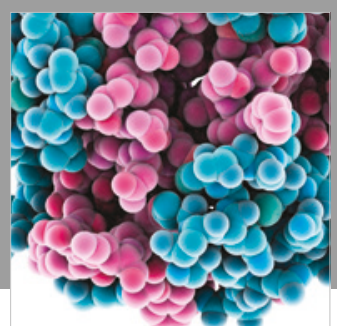

ournal of

Diabetes Research

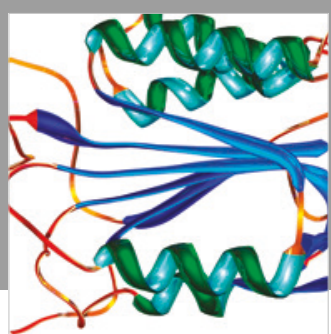

Disease Markers
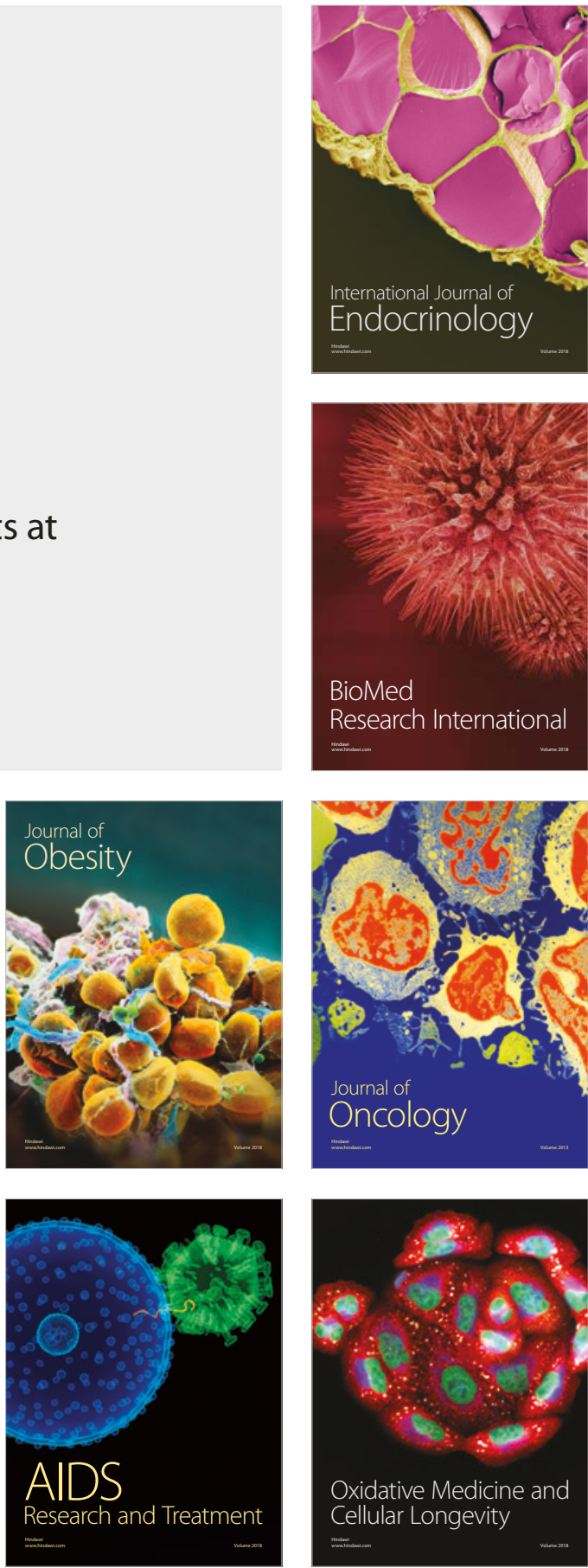